\title{
Measurement of the inclusive jet and dijet production with the ATLAS detector
}

\section{Zdenek Hubacek*}

Czech Technical University in Prague

E-mail: zdenek. hubacek@cern.ch

on behalf of the ATLAS Collaboration

\begin{abstract}
The production of inclusive jets and dijets at hadron colliders provides a stringent test of perturbative QCD at the highest energies. The process can also be used to probe the gluon density function of the proton. The ATLAS Collaboration has measured the inclusive jet production cross-section in $20.3 \mathrm{fb}^{-1}$ of data collected at a center-of-mass energy of $8 \mathrm{TeV}$ and in $3.2 \mathrm{fb}^{-1}$ of data collected at a center-of-mass energy of $13 \mathrm{TeV}$. The measurements have been performed differentially in jet rapidity and transverse momentum. The collaboration also presents a measurement of the dijet cross-section at a center-of-mass energy of $13 \mathrm{TeV}$ as a function of the dijet mass and rapiditydifference. The results have been compared with state-of-the-art theory predictions at NLO in pQCD, interfaced with different parton distribution functions. Special focus is drawn on the correlation models of the associated systematic uncertainties and the interpretation of the $\chi^{2}$-values resulting from theory comparisons. The $13 \mathrm{TeV}$ measurements have also been compared with the more recent NNLO predictions.
\end{abstract}

XXVI International Workshop on Deep-Inelastic Scattering and Related Subjects (DIS2018)

16-20 April 2018

Kobe, Japan

${ }^{*}$ Speaker. 


\section{Introduction}

Precise measurements of jet cross-sections are important in understanding the strong interaction in the framework of Quantum Chromodynamics (QCD). In QCD, quarks and gluons interact in the short-distance scattering processes. Jets are then interpreted as results of the quark and gluon fragmentation. Measurements of jet cross-sections provide information about the structure of the proton (parton distribution functions, PDFs) and properties of the QCD, like the strong coupling constant $\left(\alpha_{\mathrm{S}}\right)$.

This summary presents the measurement of the inclusive jet cross-section measured by the ATLAS Experiment [1] at the Large Hadron Collider [2] at the centre-of-mass energy of $8 \mathrm{TeV}$ [3] and the measurements of the inclusive jet and dijet cross-sections at the centre-of-mass energy of $13 \mathrm{TeV}$ [4]. The inclusive jet cross-sections are measured double-differentially as a function of the jet transverse momentum, $p_{\mathrm{T}}$, and absolute jet rapidity $|y|$. The dijet cross-sections are also measured double-differentially as a function of the dijet invariant mass, $m_{j j}$, and $y^{\star}$ which denotes half of the rapidity difference between the two selected jets with $|y|<3$.0. Next-to-leading order (NLO) perturbative QCD (pQCD) predictions are calculated using several current PDF sets. The predictions are corrected for electroweak and non-perturbative effects.

\section{Inclusive jet cross-section}

The inclusive jet cross-sections were measured in a kinematic region between $70(100) \mathrm{GeV}$ and $2.5(3.5) \mathrm{TeV}$ at a centre-of-mass-energy of $8(13) \mathrm{TeV}$, in both cases with jet rapidity $|y|<3.0$. The jets are reconstructed using the anti- $k_{\mathrm{T}}$ jet algorithm with a radius parameter $R=0.4$ and $R=$ 0.6 for the centre-of-mass-energy of $8 \mathrm{TeV}$ ( $R=0.6$ for the centre-of-mass-energy of $13 \mathrm{TeV})$. The reconstructed jet spectra in data are corrected for detector inefficiencies and resolution effects. The unfolding of the resolution effects is based on the iterative dynamically stabilized (IDS) method in which the transfer matrix constructed using samples of simulated events is inverted. The unfolding matrix depends on the details of the simulated model, this model improves when iterated where the number of iterations is chosen such as the residual bias coming from a closure test of the method is less than $1 \%$. In these measurements only one iteration is used. The statistical uncertainties are propagated through the unfolding procedure using an ensemble of pseudo-experiments.

The cross-sections measured at $8 \mathrm{TeV}$ are shown in Fig. 1 and the detailed theory to data comparison is shown in Fig. 2. The dominant experimental uncertainties are from the jet energy scale, jet energy resolution and unfolding effects. The theoretical uncertainty consists of the uncertainty in the choice of PDF, the strong coupling constant, the scale uncertainty (missing higher order corrections) and the uncertainty in the non-perturbative and electroweak effects which are applied to the parton level predictions. The comparison of experimental and theoretical uncertainties for a particular phase space is shown in Fig. 3. Additional quantitative analysis is performed using a $\chi^{2}$ study. The $\chi^{2}$ value and the corresponding observed $p$-value are computed using the asymmetries and the correlations of the experimental and theoretical uncertainties. A satisfactory description is found for all PDF sets for jets with $R=0.4$. For jets with $R=0.6$ a good description is found for jets with $|y|>1.0$, but for the central region, the agreement is worse than for $R=0.4$. 

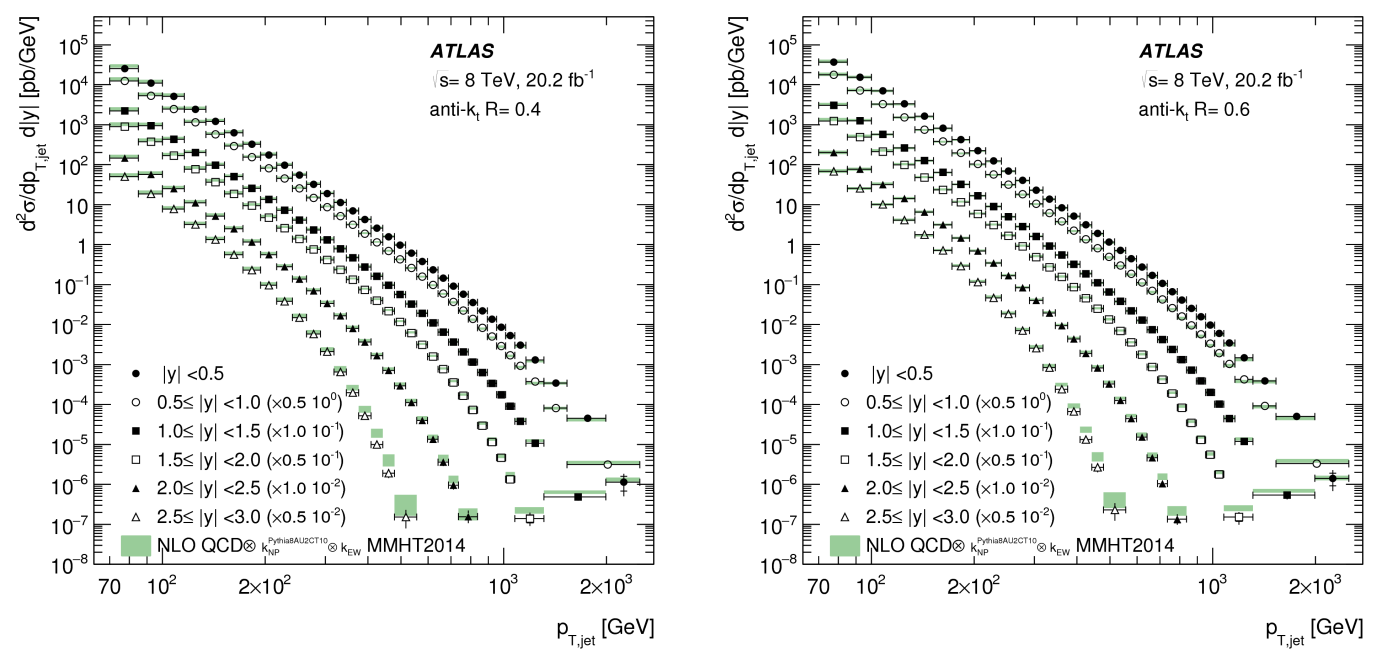

Figure 1: Inclusive jet cross-sections as a function of the jet transverse momentum $p_{\mathrm{T}}$ in 6 bins of jet rapidity at a centre-of-mass energy of $8 \mathrm{TeV}$ for jet algorithm $R=0.4$ (left) and $R=0.6$ (right). The data are shown together with the NLO prediction using MMHT2014 PDF set corrected for electroweak and nonperturbative effects [3].
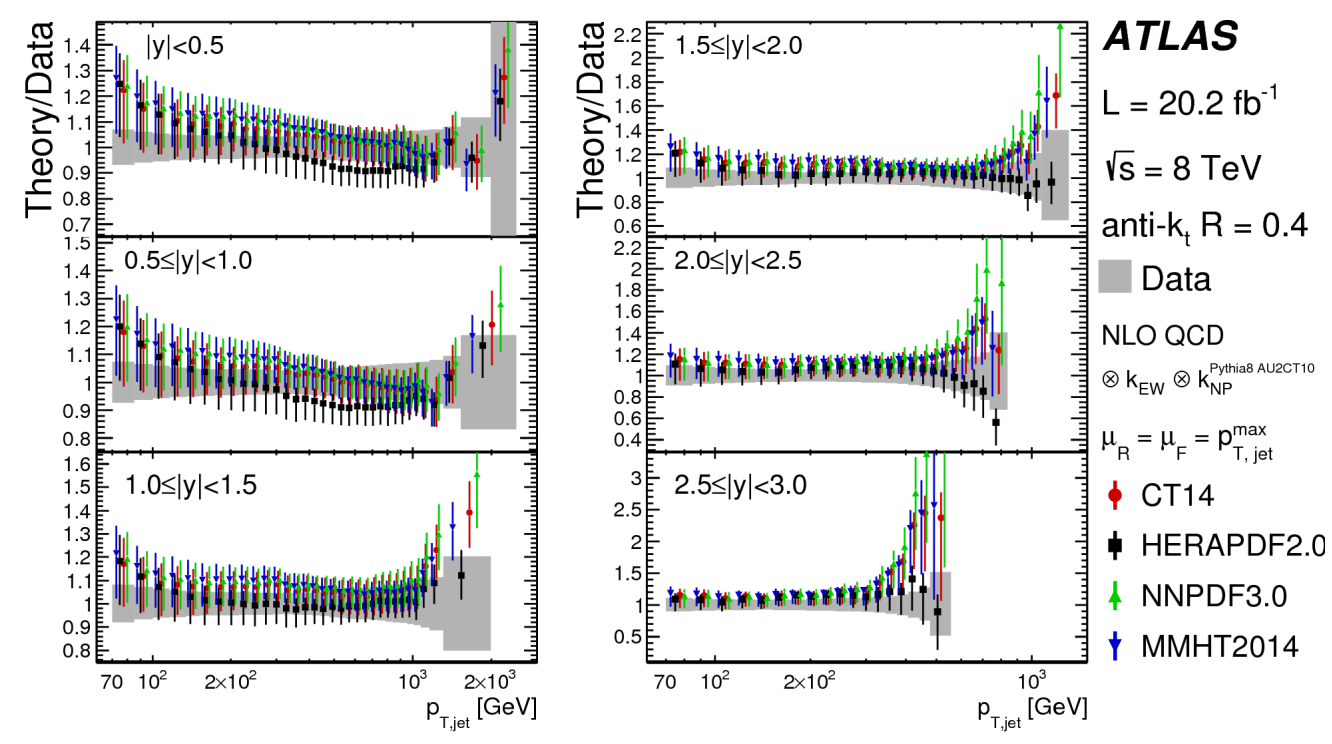

Figure 2: Ratio of the inclusive jet NLO cross-section prediction corrected for electroweak and nonperturbative effects to the measured cross-section in data for various PDF sets for jets $R=0.4$. Figure taken from [3]. 

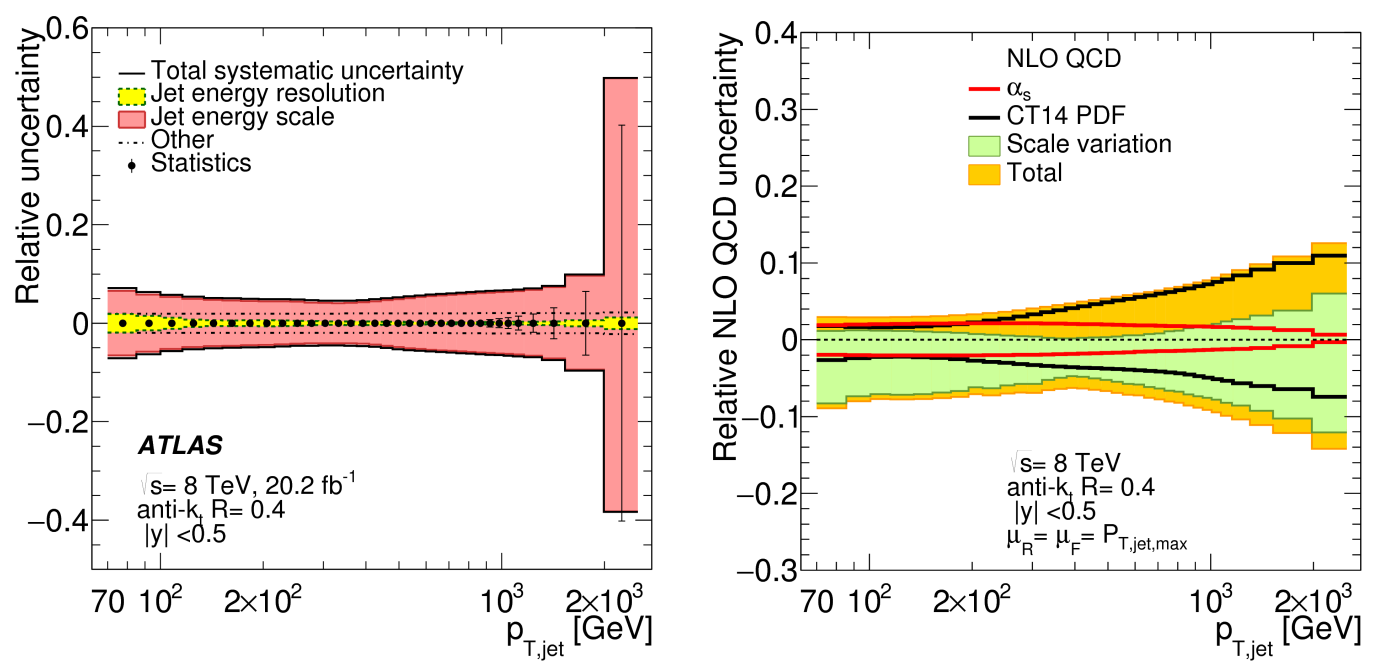

Figure 3: Example summary of the total experimental (left) and theoretical (right) uncertainties for the $8 \mathrm{TeV}$ measurement for $R=0.4$ jets and the central region $|y|<0.5$. Figures taken from [3].

The cross-sections measured at $13 \mathrm{TeV}$ are shown in Fig. 4 (left). For the first time, the detailed comparison with the next-to-next-to-leading order (NNLO) pQCD prediction is shown in Fig. 5. It shows a similar level of agreement as the NLO result, but the choice of appropriate scale is under discussion. The full quantitative analysis of the agreement is currently not possible due to computing complexity of the NNLO calculation.
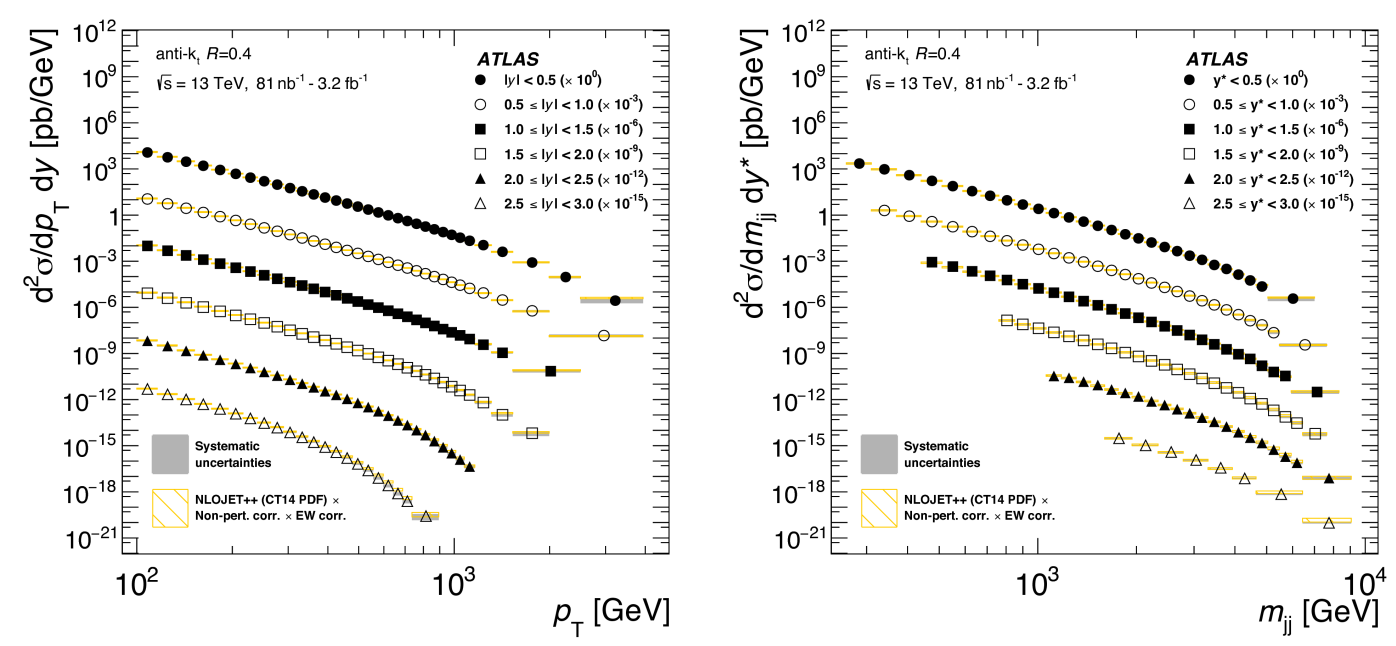

Figure 4: Inclusive jet (left) and dijet (right) cross-sections as functions of the jet transverse momentum and dijet invariant mass at a centre-of-mass energy of $13 \mathrm{TeV}$ for jets with $R=0.4$. Figures taken from [4].

\section{Dijet cross-section}

The dijet double-differential cross-section is measured as a function of the dijet invariant mass 

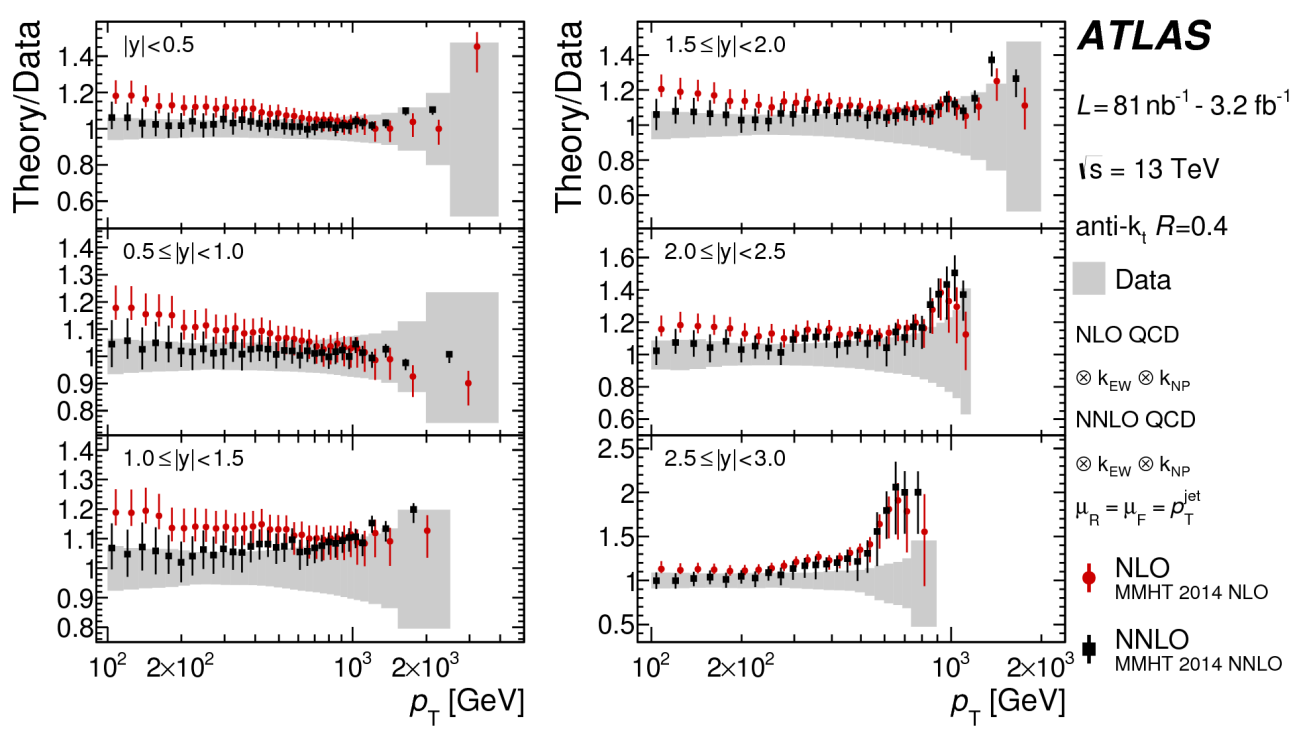

Figure 5: Ratio of the inclusive jet NNLO cross-section prediction to the measured cross-section in data for various PDF sets [4].

$m_{j j}$, in six equal-size bins of $y$, for events with at least two jets with $p_{\mathrm{T}}>75 \mathrm{GeV}$ and $|y|<3.0$ is shown in Fig. 4 (right). In addition, the scalar sum of the $p_{\mathrm{T}}$ of the first and second leading jets, $H_{T, 2}=p_{\mathrm{T}, 1}+p_{\mathrm{T}, 2}$, is required to be above $200 \mathrm{GeV}$ to avoid instabilities in the NLO cross-section calculations with the symmetric $p_{\mathrm{T}}$ requirement applied to the leading and sub-leading jets. The ratio of the dijet NLO cross-section prediction to the measured cross-section is shown in Fig. 6. The quantitative analysis shows overall a good agreement.
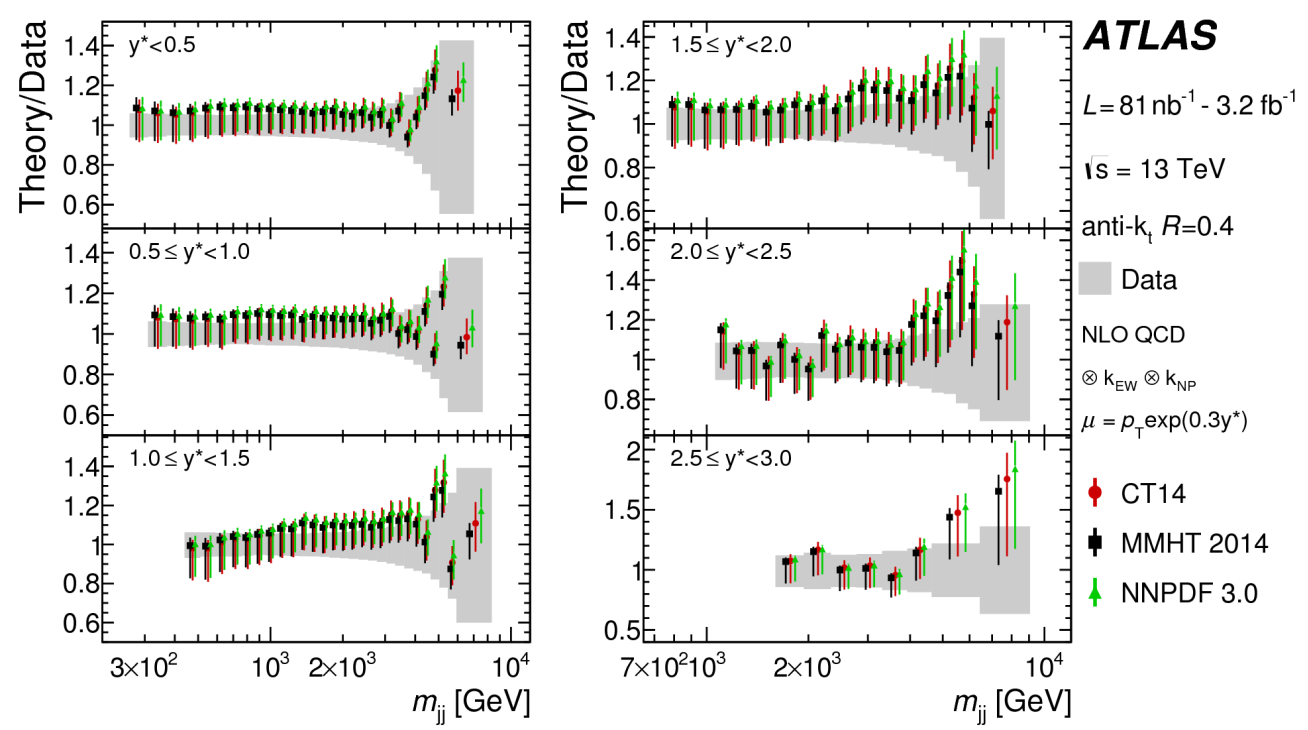

Figure 6: Ratio of the dijet NLO cross-section prediction corrected for electroweak and non-perturbative effects to the measured cross-section for various PDF sets [4]. 


\section{Summary}

The inclusive jet cross-sections were measured by the ATLAS Collaboration at the Large Hadron Collider at a centre-of-mass energy of $8(13) \mathrm{TeV}$. The inclusive jet cross-sections were measured in a kinematic region between $70(100) \mathrm{GeV}$ and $2.5(3.5) \mathrm{TeV}$ in both cases with $|y|<$ 3.0. The cross-sections are compared with the state-of-art fixed order perturbative QCD calculations. A quantitative comparison of the measurements to fixed-order NLO QCD calculations and electroweak effects, shows overall fair agreement and consistency of the two measurements. The cross-sections measured at $13 \mathrm{TeV}$ are also for the first time compared to the predictions at NNLO. The dijet cross-sections were measured at a centre-of-mass energy of $13 \mathrm{TeV}$. These cross-sections are presented as a function of the dijet invariant mass in the range between $300 \mathrm{GeV}<m_{j j}<9 \mathrm{TeV}$ for jets with $|y|<3.0$ and $y^{\star}<3.0$. The quantitative comparison shows a good agreement between the theory and the measured cross-sections.

\section{Acknowledgments}

The author gratefully acknowledges the financial support from the Ministry of Education, Youth and Sport of the Czech Republic under the Grant No. RVO 68407700.

\section{References}

[1] ATLAS Collaboration, The ATLAS Experiment at the CERN Large Hadron Collider, JINST 3 (2008) S08003.

[2] L. Evans and P. Bryant, LHC Machine, JINST 3 (2008) S08001.

[3] ATLAS Collaboration, Measurement of the inclusive jet cross-sections in proton-proton collisions at $\sqrt{s}=8 \mathrm{TeV}$ with the ATLAS detector, JHEP 09 (2017) 020 [1706 . 03192].

[4] ATLAS Collaboration, Measurement of inclusive jet and dijet cross-sections in proton-proton collisions at $\sqrt{s}=13 \mathrm{TeV}$ with the ATLAS detector, 1711.02692 . 